\section{Spatial Resolution in ACOM - What Will Come After EBSD}

\author{
R.A. Schwarzer \\ Kappstr. 65, D-71083 Herrenberg, Germany \\ mail@crystaltexture.com
}

\section{Introduction}

Automated Crystal Orientation Microscopy (ACOM) on a grain specific level has proved to be an invaluable new tool for characterizing polycrystalline materials. It is usually based on scanning facilities using electron diffraction [1], due to its high sensitivity and spatial resolution, but also attempts have been made which rely upon X-ray or hard synchrotron radiation diffraction. The grain orientations are commonly mapped in pseudo-colors on the scanning grid to construct Crystal Orientation Maps (COM), which represent "images" of the microstructure with the advantage of providing quantitative orientation contrast. In a similar way, misorientations across grain boundaries, $\Sigma$ values of grain boundaries, or other microstructural characteristics are visualized by mapping the grains in the micrograph with specific colors. The principal objectives are the determination of quantitative, statistically meaningful data sets of crystal orientations, misorientations, the CSL character $(\Sigma)$ of grain boundaries, local crystal texture (pole figures, ODF, MODF, OCF) and derived entities, phase discrimination and phase identification.

\section{ACOM based on electron diffraction}

The highest spatial resolution of orientation measurement is achieved in the TEM by high-resolution lattice imaging. However, this tedious technique has unrivalled benefits when studying misorientations or the structure of grain boundaries, but is not appropriate for routinely collecting a large number of grain orientations. Selected Area Diffraction (SAD) and in particular spot as well as Transmission Kikuchi diffraction Patterns (TKP) produced with a focused primary beam (so-called MicroBeam Diffraction, MBD) allow a fast computeraided acquisition of grain orientations down to a few nanometer spatial resolution. But then the sample foils must be equally thin so that the patterns from overlapping grains are not superimposed. Fine grains should have a columnar shape extending in the direction of the beam, usually perpendicular to the foil. Sufficient scattering of the incoming beam is required for the formation of TKP. Therefore, the foil thickness should exceed several tens of nanometers. However, large-area, uniformly-thin foils are difficult to prepare for TEM observation and, even worse, are sources of orientation errors due to wrinkling of the

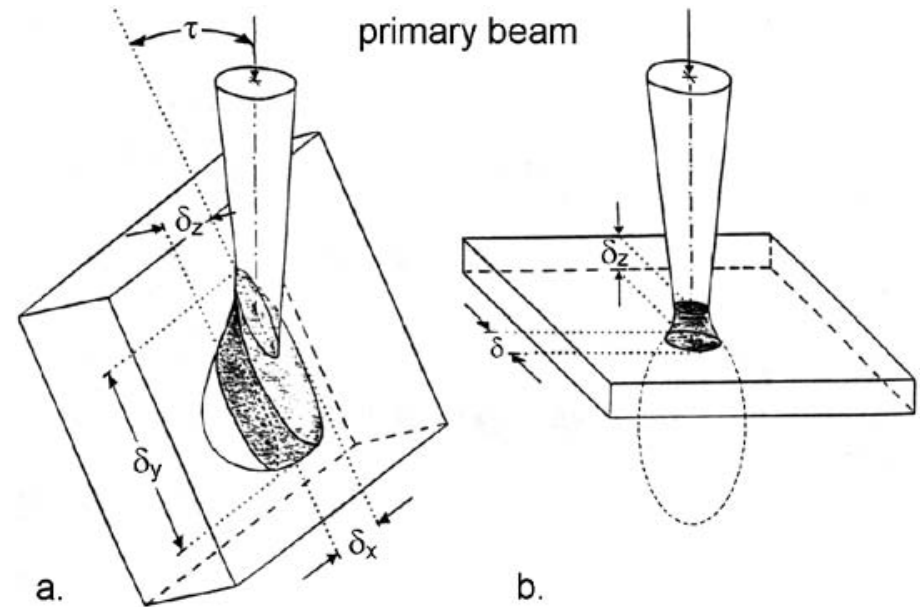

Fig. 1 Interaction volume, excitation volume and spatial resolutions, $\delta$, with a. Backscatter Kikuchi Patterns from a bulk specimen in the SEM and $b$. Transmission Kikuchi Patterns from a thin foil in the TEM. specimen manifested by bend contours in the TEM image of the foll.

ACOM on bulk surfaces without user intervention is most frequently performed in the SEM by evaluating Backscatter Kikuchi Patterns (BKP). The beam spot is digitally scanning across the specimen, one after the other BKP is acquired, transferred to the computer and indexed, and the crystal orientation of the grain under the beam spot is calculated [2]. The sample is tilted out of the horizontal to typically $70^{\circ}$ towards a phosphor screen to increase intensity for pattern collection. Spatial resolution is in the range of $<0.05 \mu \mathrm{m}$, accuracy is better than $0.5^{\circ}$, and speed of on-line measurement is actually between 30 000 and 3 million orientations per hour, depending on the hardware. This technique is also known as "Electron BackScatter Diffraction" (EBSD) if referred to the commercial trademark systems OXFORD/ HKL "Channel" or EDAX/TSL "OIM".

A high spatial resolution in orientation measurement is required for the study of fine grained and heavily deformed materials in general, of recrystallization and grain growth, of grain boundary characterization, of nanomaterials and in the study of the impact effects of high energy particles. But why does spatial resolution in EBSD fall more than one order of magnitude behind spatial resolution in conventional SEM imaging, and behind still further when compared to the spatial resolution of a TEM? The inherent resolution of EBSD is based primarily not upon the diameter of the beam spot at the point of impact on the surface, but by the excitation volume. That is the fraction of the interaction volume of the primaries with the sample from where the pattern forming electrons are back-diffracted and can leave the crystal without further scattering. This volume fraction is indicated by shading in Fig 1. As a consequence of the steep sample tilt, elongated projection of the beam spot and forward scattering, spatial resolution in EBSD along the beam direction on the sample surface, $\delta_{y}$, is about three times worse than $\delta_{\mathrm{x}}$. The information depth, $\delta_{z}$, is limited by the

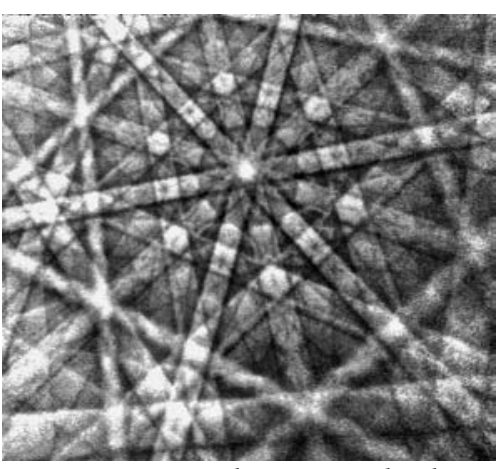

Fig. 2 Backscatter Kikuchi Pattern from cadmium at $20 \mathrm{keV}$. mean free depth of penetration of the backdiffracted electrons in the sampled material at the actual beam voltage. The excitation volume increases for light materials and high beam voltages. The TEM is operated at a significantly higher accelerating voltage than the SEM. However, spatial resolution, $\delta$, in microbeam TEM diffraction is still approximately the diameter of the beam size, because the sample has to be thinned to a thickness in the range of the mean free path of the energetic electrons so that only a small interaction volume can develop (Fig. 1b). Information depth, $\delta_{z}$, equals foil thickness. This makes the point that for ACOM in a SEM it is not wise to reduce the spot size below the diameter of the excitation volume. The adverse effects would be a reduced beam current, hence less intense patterns, and possibly a strong increase in contamination rate by polymerization of hydrocarbons under the beam.

Spatial resolution in EBSD can be improved to some extent by lowering the beam voltage from typically $20 \mathrm{keV}$ down to a few $\mathrm{keV}$. However, source brightness and sensitivity of the phosphor screen, hence pattern intensity are likewise reduced. While resolution within a grain is of low significance, it becomes quite critical when the beam approaches a grain boundary. A smart pattern indexing software program can then improve resolution by taking account of the intensity levels of 
superimposed patterns, rejecting less likely orientation solutions and comparing orientations in neighboring pixels.

\section{Ion blocking patterns}

Diffraction patterns are produced not only by electrons but also by ions of a few ten keV kinetic energy when impinging on a crystalline surface. They have been recorded on special photographic plates, which cover a large solid angle and are called Ion Blocking Patterns (IBP) [3, 4]. As a consequence of the much shorter deBroglie wavelength and the specific interaction of ions with solid crystals, IBP have, at a first
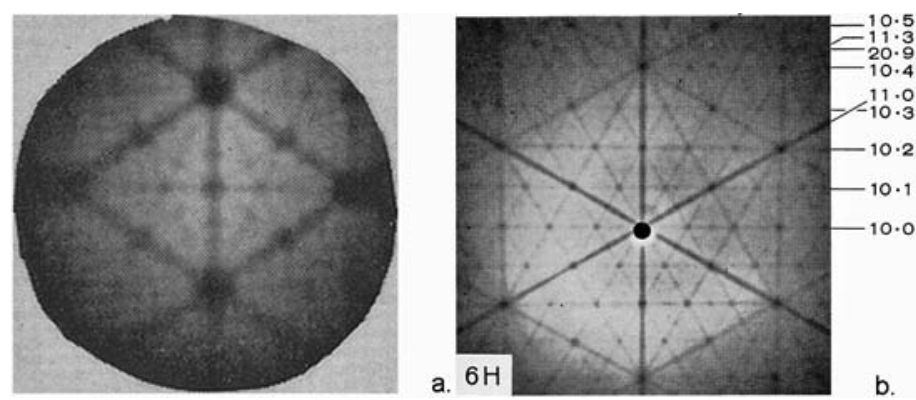

Fig. 3 Ion blocking patterns a. from a (110) face when a $20 \mathrm{keV}$ proton beam incident on a tungsten single crystal (After Nelson 1968 [3] Fig. 5) and $\mathrm{b}$. from hexagonal $\mathrm{SiC}$ for $150 \mathrm{keV}$ protons incident in normal direction on the (00.1) single crystal face. The black dot in the center marks the hole in the film for letting the primary beam pass through (After Barrett et al., 1970 [5] Fig. 1). In both set-ups the photographic plates were positioned substantially parallel to the specimen surface.

glimpse, an appearance substantially different from BKP. Instead of broad Kikuchi bands (Fig. 2), they show narrow straight black bands, almost lines, with a much higher contrast on a flat background (Fig. 3 ). However, the geometry of IBP and BKP is quite similar. The center line of a band corresponds to the section line of the - imaginarily extended - diffracting lattice plane with the recording plane, and the crossing points of bands, named poles, represent the positions of zone axes of the crystal. So the crystal structure and crystal orientation of the diffracting volume can be determined from the intensity distribution and positions of the bands in an IBP [5] in quite a similar way as in a BKP.

Intense focused ion beams are produced with liquid metal ion sources or with gas field ionization sources [6]. An outstanding high source brightness of more than $10^{9} \mathrm{~A} / \mathrm{cm}^{2} \mathrm{sr} \mathrm{He}{ }^{+}$ions has been achieved by using a $<111>$ oriented sharp field emitter tip of tungsten [7] so that beam currents up to $100 \mathrm{pA}$ at an energy spread of less than $1 \mathrm{eV}$ and a beam voltage of typically 20 to $30 \mathrm{kV}$ are practical [8]. Since in addition, both the chromatic as well as the diffraction aberrations of the probe forming lens are less affecting in this set-up, it is expected that it will be possible to focus the beam from the field ionization source into a probe size down to the sub-nanometer range. Thanks to the small excitation volume, a similar high spatial resolution seems possible in ion imaging microscopy and backscatter ion diffraction that is significantly much smaller than in the SEM. Ion induced secondary electrons as well as backscattered ions are used for imaging the specimen surface in the scanning ion microscope. An excellent orientation contrast is obtained from polycrystalline surfaces [9]. This is a clear indication of the strong blocking effect [3]: When the ion beam impinges on the specimen, it is fanned out over large angles due to the onset of inelastic scattering. Further propagation of the ions in the crystal depends on their direction of movement with respect to the lattice. Ions that propagate at angles wider than certain classical critical angles of incidence to densely packed lattice planes or rows of atoms are blocked and backscattered. At certain critical angles with respect to the lattice, the ions are channeled deeper in the crystal. Hence a low signal of backscattered ions as well as secondary electrons is detected at these angles. This classical ballistic model of channeling, based on the assumption of Rutherford scatter- ing, describes the positions of bands in IBP quite well. The quantum mechanical treatment of ion diffraction has to consider a multiple beam approach [10]. The results correspond to those of the classical treatment, in particular that the band width of IBP is within the mass invariant limit of the critical angle, whereas the width of a Kikuchi band in electron diffraction is given by twice the Bragg angle.

For recording the $2 \mathrm{D}$ intensity distribution of the backscattered ions, i.e. the ion blocking pattern, an imaging ion detector is required. Ion-sensitive photographic plates are an impractical recording medium for ACOM. Phosphor screens are a proven means for recording electron diffraction patterns. They can be viewed through a window from outside or imaged with a video camera. However, they are damaged by ion bombardment so that sensitivity will drop after a short period of exposure to ion blocking patterns. For long-term operation, an open microchannel plate can serve as an ion-to-electron image converter (Figure 4). When an ion hits the wall of a microchannel, secondary electrons are released that are accelerated in the electric field to the exit side of the plate. These secondaries may also impinge on the channel walls and set free further secondaries so that finally a cascade of electrons per incoming ion will leave the microchannel on its exit side and excite the phosphor screen. This amplification of the signal by releasing more than one electron per incident ion is beneficial, but not strictly necessary for image conversion. It is advisable, however, to maintain the entrance side of the channel plate at a low negative potential to keep away low energy secondary electrons that have been set free by ion impact from the sample and the walls of the specimen chamber. The secondary electrons have a smooth angular intensity distribution without useful information of the IBP geometry and would otherwise increase background. The low electric field between specimen and channel plate does not affect high energy ions. A fraction of the backscattered ions can lose their charge after diffraction in the crystal and on the way to the detector, but without significantly changing the direction of movement. These high energy neutrals still bear the information of IBP, but they could pass through the channel plate and damage a phosphor screen behind. The neutrals can be utilized with profit to increase the intensity of the converted electron image of the IBP, if one more channel plate is placed before the first one whereby their microchannel directions are slightly inclined against each other ("chevron type channel plates") as to intercept the direct pass of neutrals.

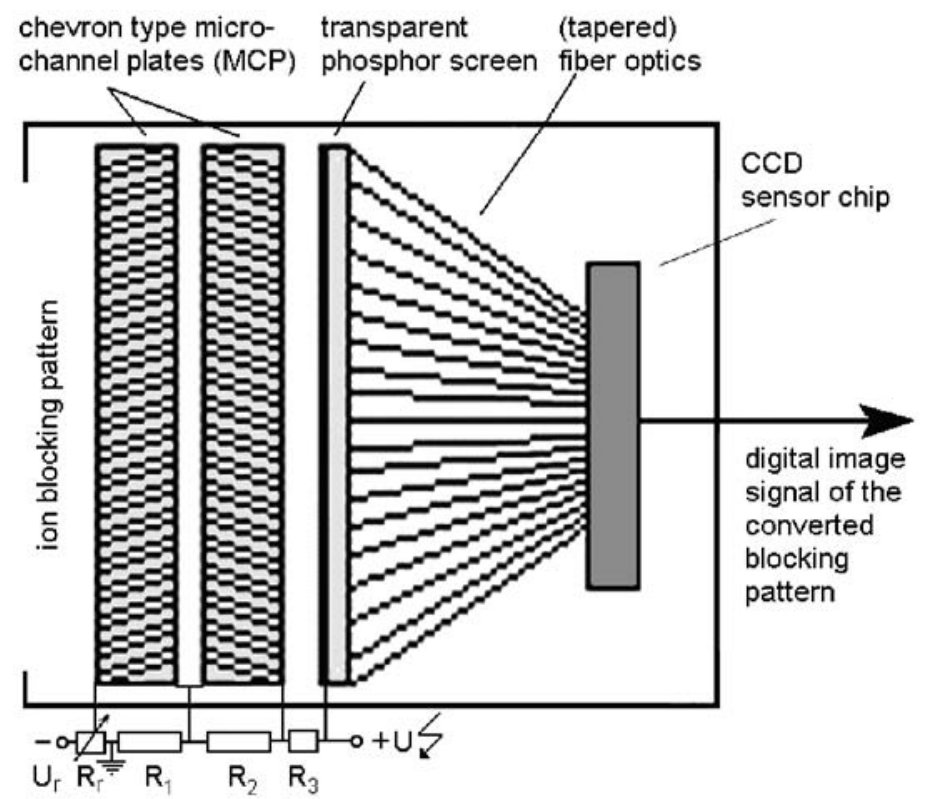

Fig. 4 Imaging ion-to-electron converter based on a chevron set of microchannel plates and a fiber-optically coupled CCD sensor. 


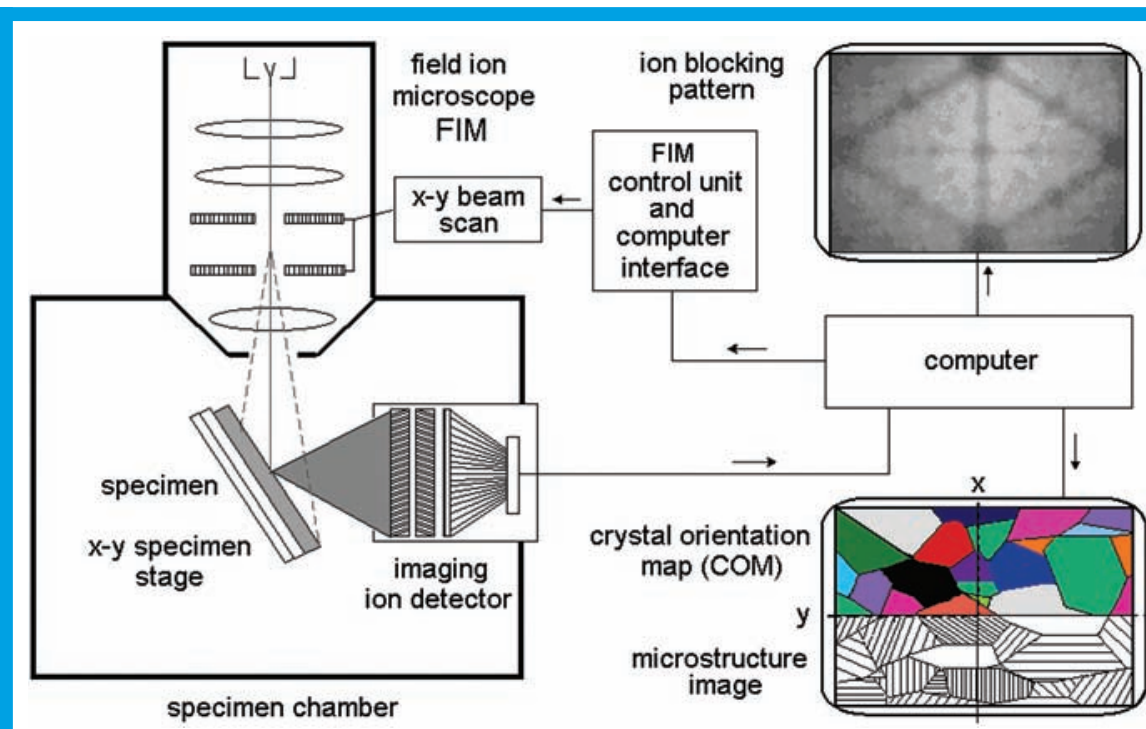

Fig. 5 ACOM with a computer-controlled ion scanning microscope using ion blocking patterns for grain orientation measurement.

Energetic neutrals will release secondary electrons in the same way as ions when they impinge on the wall of a channel. The converted IBP is projected on a phosphor screen and recorded with a (fiber-optically coupled) CCD camera.

\section{Advantages of ion blocking diffraction over electron back- scatter diffraction}

A similar technique as for BKD [2] can be used for the acquisition and indexing of IBP. Allowance has to be made in the software for the different background intensities, pattern contrast and profiles of the Kikuchi bands vs. the blocking bands. A schematic set-up of ACOM with IBP and a focused ion beam is shown in Fig. 5 [11]. It promises the following advantages over EBSD in the SEM:

- It can be technically realized as an accessory to an ion scanning microscope.

- The sample is tilted at moderate angles of about $45^{\circ}$ to the primary beam direction to accommodate the wide-angle pick-up of IBP. So image distortion and spatial resolution in beam direction are markedly reduced as compared to EBSD where the specimen is steeply tilted to typically $70^{\circ}$.

- Sample preparation is less difficult since deformation layers or foreign surface layers can be removed at a controlled rate in situ by using a primary beam of heavy ions until clear blocking patterns have developed.

- $3 \mathrm{D}$ reconstruction of the volumetric microstructure from planar $2 \mathrm{D}$ slices is facilitated by controlled serial ion sectioning. A smooth surface that shows little damage is produced at shallow incidence of the beam. For ACOM the specimen may remain stationary in this position or is simply tilted to a steeper angle to the beam if a higher spatial resolution is required in ion blocking diffraction. In a combined SEM-FIB system, on the contrary, the specimen has to be realigned for grazing incidence FIB surface polishing requiring subsequent delicate tilt-rotation movement of the specimen to return to the EBSD beam position.

- Specimen charging is less harmful than in the SEM because secondary electrons are released from walls of the specimen chamber by the impact of scattered ions and neutrals. They reduce positive surface charging.

Implementation of ACOM in a Helium Ion Microscope $[8,12]$ affords particular advantages:

- A gas field ionization source can be operated with a variety of ionization gases, for example hydrogen, oxygen, nitrogen and noble gases [13], whereas a liquid metal ion source is limited to produce one species of ions (e.g. $\mathrm{Ga}^{+}$or $\mathrm{In}^{+}$) only.

- When using a primary beam of light ions, as for instance protons or $\mathrm{He}^{+}$, virtually no specimen sputtering is introduced during orientation measurement nor during imaging. The contamination rate is low.

- The excitation volume is not significantly larger than the minimum spot size. Spatial and in-depth resolution of ACOM with IBP is expected to approach the sub-nanometer range.

- By alternating between a beam of heavy and light ions, a sequential in-depth investigation is performed whereby the specimen is maintained stationary (3D crystal orientation mapping).

- Specimens that adversely react to $\mathrm{Ga}^{+}$ions, such as aluminum based alloys, can be investigated.

\section{Conclusion}

EBSD has become the standard technique for automated crystal orientation microscopy on bulk polycrystals at a grain specific level. Reasons are easy operation of commercial BKD systems and the wide availability of SEM instruments. The study of fine grain and heavily deformed materials in general, of nanomaterials, of recrystallization, grain growth and grain boundary characterization demands a substantially higher spatial resolution than is achieved with EBSD in the SEM. The combination of ion scanning microscopy and ion blocking diffraction promises a high spatial and depth resolution down to the sub-nanometer range in imaging and diffraction mode.

\section{Acknowledgment}

The IBP in Fig. 3a is reprinted from [3] (C) 1967, Taylor \& Francis, and the IBP in Fig. 3b is reprinted from [5] (c) 1970, American Institute of Physics. The permissions for reproduction are acknowledged.

\section{References}

[1] R.A. Schwarzer: The determination of local texture by electron diffraction - a tutorial review. Textures and Microstructures 20 (1993) 7-27.

[2] R.A. Schwarzer: Automated crystal lattice orientation mapping using a computer-controlled SEM. Micron 28 (1997) 249-265.

[3] R.S. Nelson: Proton scattering microscopy. Phil. Mag. (8) 15 (1967) 845-854

[4] R.G. Livesey: A $30 \mathrm{keV}$ instrument for ion surface interaction studies. Vacuum 22 (1972) 595-597.

[5] C.S. Barrett, M.A. Barrett, R.M. Mueller and W. White: Identification of silicon carbide phases by proton blocking patterns. J. Appl. Phys. 41 (1970) 2727-2728.

[6] V.N. Tondare: Quest for high brightness, monochromatic noble gas ion sources. J. Vac. Soc. Techn. A 23 (2005) 1498-1508.

[7] B.W. Ward, L. Farkas, J.A. Notte, and R.G. Percival: Systems and methods for a gas field ionization source. US Patent 2007/0228287 A1.

[8] J. Morgan, J. Notte, R. Hill and B. Ward: An introduction to the helium ion microscope. Microscopy Today 14\#4 (2006) 24-31.

[9] U. Wenck and G. Nolze: FIB milling and channeling. GIT Imaging and Microscopy 9/3 (2007) 34-36.

[10] L.T. Chadderton: A correspondence principle for the channelling of fast charged particles. Phil. Mag. (8) 18 (1968) 1017-1031.

[11] R. Schwarzer: Patent pending.

[12] L. Scipioni, L. Stern and J. Notte: Applications of the helium ion microscope. Microscopy Today 15\#6 (2007) 12-15.

[13] R. Schwarzer and K.H. Gaukler: Erzeugung einer Ionen-Mikrosonde mittels Feldionisation und Emissionslinse. Vakuum-Technik 27 (1978) 2-5. 